DOI 10.1007/s 10812-016-0203-7

Journal of Applied Spectroscopy, Vol. 82, No. 6, January, 2016 (Russian Original Vol. 82, No. 6, November-December, 2015)

UDC 621.315.592;535.37

\title{
EFFECT OF SUBSTITUENTS IN POLYVINYLCARBAZOLE STRUCTURES ON THEIR OPTICAL PROPERTIES
}

\author{
Ya.Vertsimakha ${ }^{1}$ P.Lutsyk ${ }^{1}$,V.Syromyatnikov' ${ }^{2}$ I.Savchenko ${ }^{2 *}$
}

(1) Institute of Physics, NASU, prosp. Nauky 46, 03680, Kyiv, Ukraine

(2) Macromolecular Chemistry Department, Taras Shevchenko National University of Kyiv, 64 Volodymyrska str., 01601, Kyiv, Ukraine

Absorption, photoluminescence, and photoluminescence excitation spectra of solutions and thin films of $N$-vinylcarbazole polymers and copolymers with various substituents directly on the carbazole moiety and on the polymer chain were studied comprehensively. Polymers that were used previously to develop polymer composites with polymethine dyes having photosensitivity over a broad spectral range including the visible and near-IR regions were selected for the studies.

Keywords:

photoluminescence, photoluminescence excitation and absorption spectra, $N$-vinylcarbazole, copolymer, octylmethacrylate, excimer

*To whom correspondence should be addressed. E-mail: iras@univ.kiev.ua

Translated from Zhurnal Prikladnoi Spektroskopii, Vol. 82, No. 6, pp. 831-835, NovemberDecember, 2015. 
Introduction. Poly(N-vinylcarbazole) has been extensively studied over the last 50 years, i.e., since its photoconductivity was discovered [1]. Many carbazole-containing polymers were synthesized and shown to possess good photorefractive, optical, and charge-transfer properties [2]. The development based on them of plastic composites that are photosensitive over a broad spectral range [3-5], organic LEDs [6], information recording materials [7], organic solar cells [8], and multi-layered structures [9] has recently stimulated considerable interest in them. Nevertheless, their electronic absorption spectra are insufficiently studied. The effects of substituents in the polymer structures on photoluminescence (PL) excitation spectra of films of their derivatives are especially poorly studied $[10,11]$.

The goal of the present work was to investigate the effects of substituents in structures of $\mathrm{N}$ vinylcarbazole polymers and copolymers on their optical properties in order to develop methods for increasing further the photosensitivity of these polymer composites with dyes.

Experimental. Polymers and copolymers of N-vinylcarbazole with octylmethacrylate (VC-OMA), $\mathrm{N}$-vinyl-3-iodocarbazole with octylmethacrylate (I-VC-OMA), N-vinyl-7H-benzo[c]carbazole (V7BC), and its copolymer with OMA (V7BC-OMA) that were soluble in volatile solvents were synthesized and purified by reprecipitation from appropriate organic solvents at the Department of Polymer Chemistry, Kyiv National University:

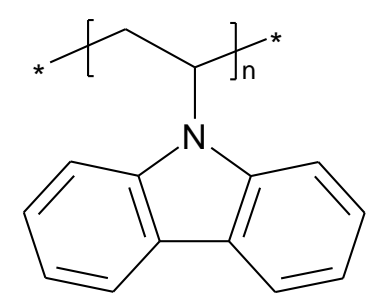

Polyvinylcarbazole (PVC)

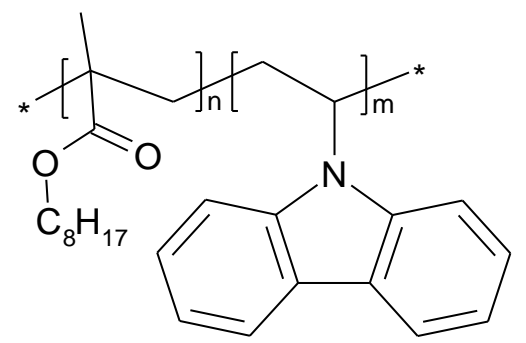

N-Vinylcarbazoleoctylmethacrylate copolymer

(VC-OMA)

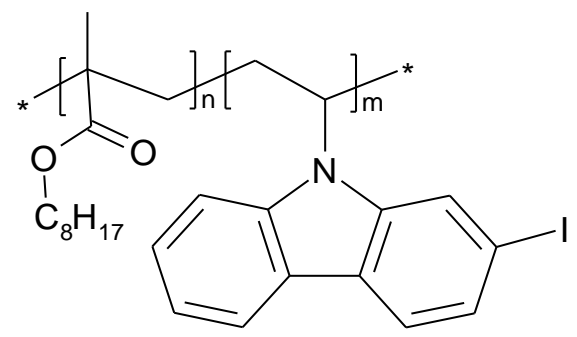

$\mathrm{N}-$ Vinyl-3-iodocarbazole with octylmethacrylate copolymer (I-VC-OMA) 


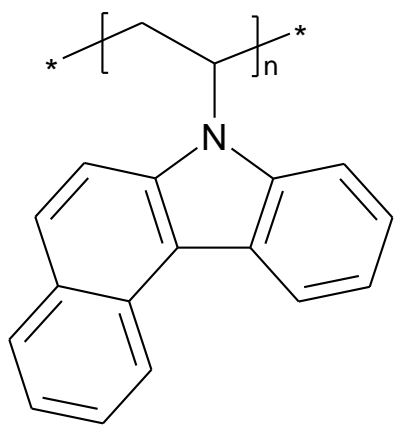

Poly(N-vinyl-7Hbenzo(c)carbazole) (V7BC)

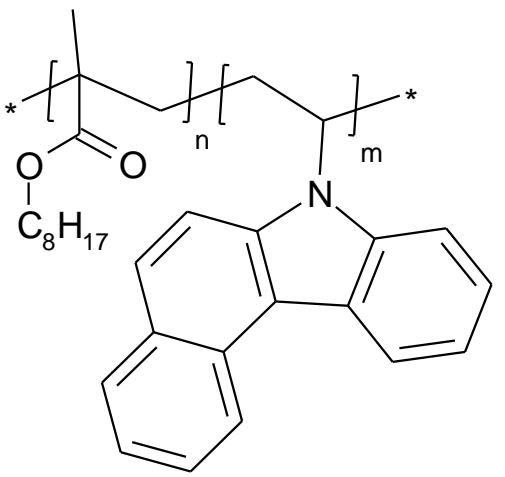

N-vinyl-7H-benzo(c)carbazole OMA copolymer (V7BC-OMA)

PVC and V7BC were polymerized and VC-OMA, V7BC-OMA, and I-VC-OMA were copolymerized in the presence of azobisisobutyronitrile initiator in $\mathrm{i}-\mathrm{PrOH}$ or toluene solution at $80 \mathrm{oC}$ under Ar for 1-2 h. Polymers were precipitated in $\mathrm{MeOH}$. The purity of the polymers was checked by reprecipitating their benzene or dioxane solutions in $\mathrm{MeOH}$. Thin films of polymers 30$100-\mathrm{nm}$ thick were prepared by pouring a solution of the polymer in $\mathrm{CHCl} 3$ onto quartz substrates.

Absorption spectra of the solutions and fi lms were measured using Shimadzu UV2450 and UV-Vis spectrophotometers. PL and PL excitation spectra were measured using a Jobin Yvon NanoLog spectrophotometer with the samples and detector placed on the same side of the fi $1 \mathrm{~m}$, i.e., with frontal illumination at an angle of $30^{\circ}$.

Results and Discussion. According to the literature [11], $\pi-\pi^{*}$-transitions ${ }^{1} \mathrm{~A} \rightarrow{ }^{1} \mathrm{~L}_{\mathrm{b}}$ and ${ }^{1} \mathrm{~A} \rightarrow{ }^{1} \mathrm{~L}_{\mathrm{a}}$ appear at $3-4 \mathrm{eV}$ in the absorption spectrum of PVC in hexane. Absorption spectra of $\mathrm{CHCl} 3$ solutions and fi lms of the synthesized polymers that were measured by us showed that the change of state (transition from solution to fi $1 \mathrm{~m})$ caused a slight red shift $(8-10 \mathrm{meV})$ of the absorption band maxima without changing the ratio of their intensities. This indicated that all studied polymers and copolymers interacted weakly in the fi lms. Therefore, solution absorption spectra are given further for simplicity (energies of band maxima were measured to an accuracy of $5 \mathrm{meV})$.

Comprehensive measurements of the absorption and PL of PVC fi lms showed that absorption maxima of the ${ }^{1} \mathrm{~A} \rightarrow{ }^{1} \mathrm{~L}_{\mathrm{b}}$-transition and PL excitation practically coincided (Fig. 1). The PL 
spectrum was independent of excitation wavelength. The PL excitation signal was negligible for hv $>3.8 \mathrm{eV}$ (near the second electronic transition), i.e., luminescence was emitted from the lower excited electronic state [10] and light quanta absorbed near the second electronic transition practically did not contribute to the PVC PL spectrum. The large bandwidth $(>1 \mathrm{eV})$ of the PL maximum at $3.10 \mathrm{eV}$, its asymmetry, and an infl ection near $3.26 \mathrm{eV}$ (Fig. 1) indicated that it consisted of several bands. Such bands were clearly visible in PL spectra of PVC-OMA and V7BCOMA fi lms (Figs. 2 and 3). This was related to the good plasticity of PVC-OMA and V7BC-OMA, which decreased the number of defects in their films.

The large Stokes shift $(0.5 \mathrm{eV})$ of the band with a maximum at $3.10 \mathrm{eV}$ (Table 1) and the lack of absorption by the films in this spectral region indicated that this was an excimer band and that there was a high probability of forming excimers during formation of the PVC fi $\mathrm{lm}$. The monomer fl uorescence band was probably located in the range 3.1-3.3 eV [10]. It was signifi cantly weakened in spectra of the studied PVC films. PVC characteristically forms several PL excimer centers (so-called sandwich-type or weakly bound excimers) with various PL emission energies [12].

A comparison of absorption by PVC (Fig. 1) and VC-OMA (Fig. 2) fi lms showed that adding the fl exible OMA chains via copolymerization with VC caused a slight shift (up to $10 \mathrm{meV}$ ) of the VC-OMA band maxima into the UV region (Table 1). This may have been due to a slight decrease of the intermolecular interaction. The PL excitation band maximum was practically the same as that of the ${ }^{1} \mathrm{~A} \rightarrow{ }^{1} \mathrm{~L}_{\mathrm{b}}$-transition.

The effect of OMA was more clearly evident in PL spectra (Fig. 2). The structuring of the PL spectrum increased (two band maxima at 3.17 and $3.34 \mathrm{eV}$ and an infl ection near $3.55 \mathrm{eV}$ appeared) (Table 1). The increased structuring of the PL band was most probably caused by the smaller bandwidth of the excimer emission as a result of the improved structure of the polymer aggregates and decreased scattering at structure defects in the fi lms. This was clearly observed even visually by comparing the morphologies of the PVC and VC-OMA fi lms. The VC-OMA films were more uniform and scattered less incident light. This could be explained by the increased plasticity of the fi lms and was confi rmed experimentally by the increased solubility of VC-OMA in organic solvents. 
TABLE 1. Band Maxima (eV) in Absorption, Photoluminescence, and Photoluminescence Excitation Spectra of Carbazole-Containing Polymer Films and Their Shifts $(\Delta \mathrm{E}, \mathrm{eV})$ Relative to the Corresponding PVC Bands (in Parentheses)

\begin{tabular}{|c|c|c|c|c|}
\hline PVC & VC-OMA & I-VC-OMA & V7BC & V7BC-OMA \\
\hline$E_{\max }$ & $\mathrm{E}_{\max }(\Delta \mathrm{E})$ & $\mathrm{E}_{\max }(\Delta \mathrm{E})$ & $\mathrm{E}_{\max }(\Delta \mathrm{E})$ & $\mathrm{E}_{\max }(\Delta \mathrm{E})$ \\
\hline \multicolumn{5}{|c|}{ Absorption spectra } \\
\hline 3.60 & $3.61(+0.01)$ & $3.52(-0.08)$ & $3.38(-0.22)$ & $3.35(-0.25)$ \\
\hline 3.76 & $3.77(+0.01)$ & $3.67(-0.10)$ & $3.55(-021)$ & $3.53(-0.23)$ \\
\hline 3.89 & - & $3.81(-0.08)$ & - & $3.77(-0.12)$ \\
\hline \multicolumn{5}{|c|}{ Photoluminescence excitation spectra } \\
\hline \multirow{3}{*}{3.58} & \multirow{3}{*}{$3.56(-0.02)$} & $3.50^{\mathrm{a}, \mathrm{b}}$ & - & $3.35(-0.23)$ \\
\hline & & $3.78(+0.2)$ & - & $3.52^{\mathrm{a}}$ \\
\hline & & & $3.78(+0.20)$ & $3.69^{\mathrm{a}}$ \\
\hline \multicolumn{5}{|c|}{ Photoluminescence spectra } \\
\hline & $3.55^{a, b}$ & & & $3.30^{\mathrm{a}}$ \\
\hline $3.26^{b}$ & $3.34(+0.08)$ & $3.32(+0.06)^{b}$ & $3.0(-0.26)$ & $3.14(-0.12)$ \\
\hline 3.10 & $3.17(+0.07)$ & $3.17(+0.07)$ & $2.56(-0.54)^{b}$ & $2.97(-0.13)$ \\
\hline
\end{tabular}

${ }^{a}$ Corresponding bands not observed in PVC spectra.

${ }^{\mathrm{b}}$ Band energy near spectral inflections. 


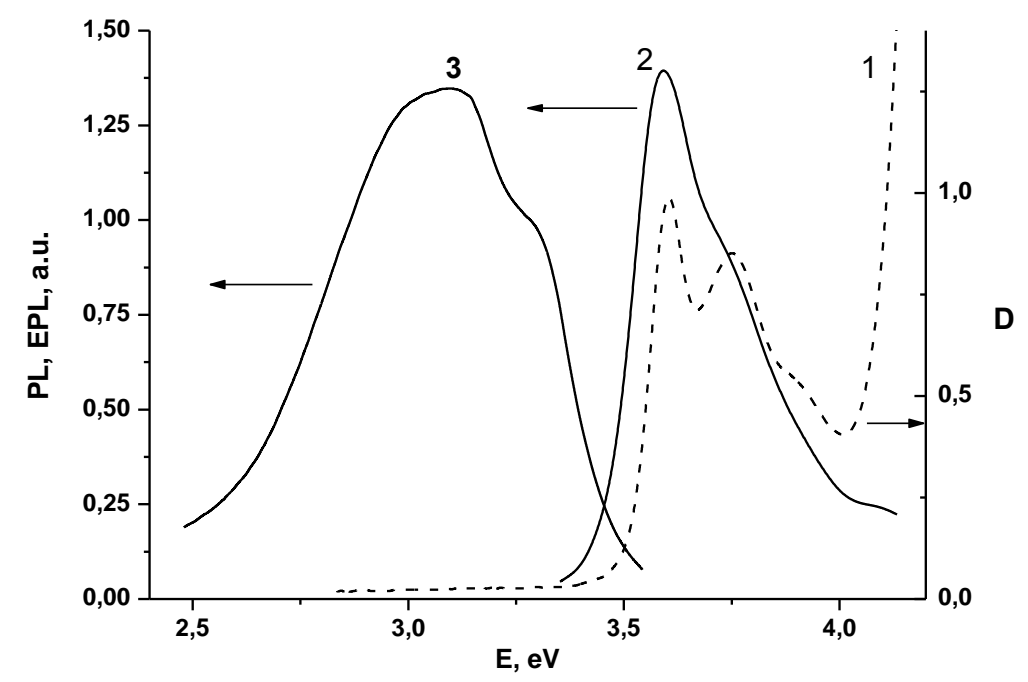

Fig. 1. Absorption spectra of PVC solution (1) and photoluminescence excitation (2) and photoluminescence (3) spectra of PVC films.

However, introducing iodine into PVC caused significant long-wavelength shifts $(80 \mathrm{meV})$ of the absorption bands and a short-wavelength shift (200 meV) of the PL excitation maximum (Table 1). This could be explained by the increased emission efficiency of the molecules, which were polarized along the $a$ axis. The number of structure defects and the light scattering at them increased simultaneously, which broadened the PL spectrum (two maxima appeared, Fig. 2). As a result, the shape of the I-VC-OMA PL band was similar to that of PVC (Fig. 1) although its maximum was shifted by $70 \mathrm{meV}$ to higher values (Table 1 ).

V7BC was constructed by adding a benzene ring to VC. This caused a substantial shift $\Delta \mathrm{E}$ of the energy of the lowest electronic ${ }^{1} \mathrm{~A} \rightarrow{ }^{1} \mathrm{~L}_{\mathrm{a}}$-transition to long wavelength (up to $250 \mathrm{meV}$ ) compared with the shift upon adding iodine to PVC $(80 \mathrm{meV})$. The absorption intensity (probability) of the ${ }^{1} \mathrm{~A} \rightarrow{ }^{1} \mathrm{~L}_{\mathrm{a}}$-transition at $3.38 \mathrm{eV}$ increased and became practically twice that of the ${ }^{1} \mathrm{~A} \rightarrow{ }^{1} \mathrm{~L}_{\mathrm{b}^{-}}$ transition (Fig. 3). The PL excitation band maximum of V7BC films was practically the same as the band maximum of the ${ }^{1} \mathrm{~A} \rightarrow{ }^{1} \mathrm{~L}_{\mathrm{a}}$-transition. 


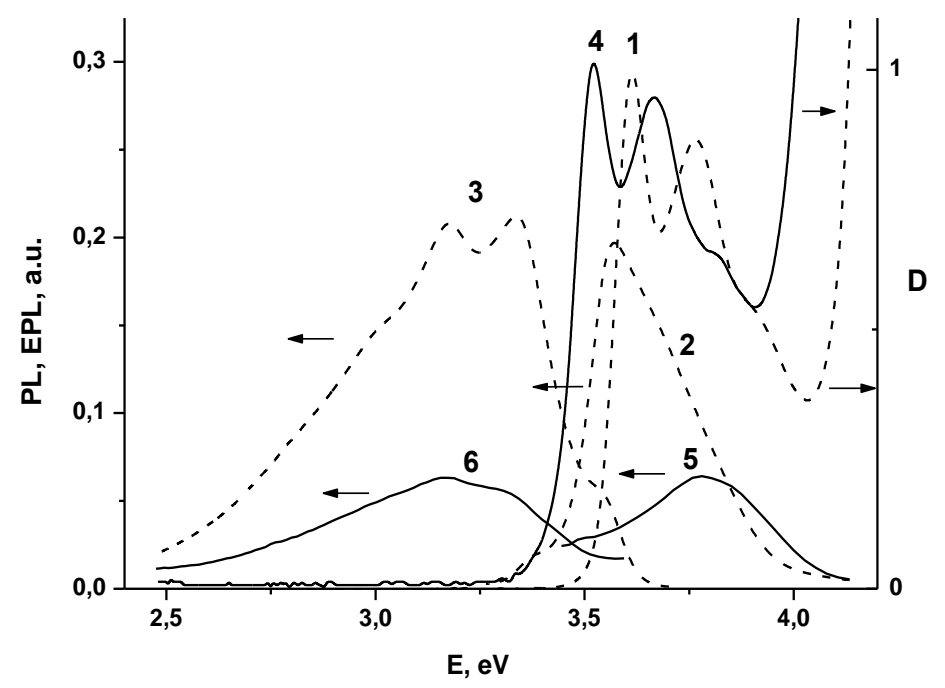

Fig. 2. Absorption spectra of solutions $(1,4)$ and photoluminescence excitation $(2,5)$ and photoluminescence $(3,6)$ spectra of films of VC-OMA (1-3) and I-VC-OMA (4-6).

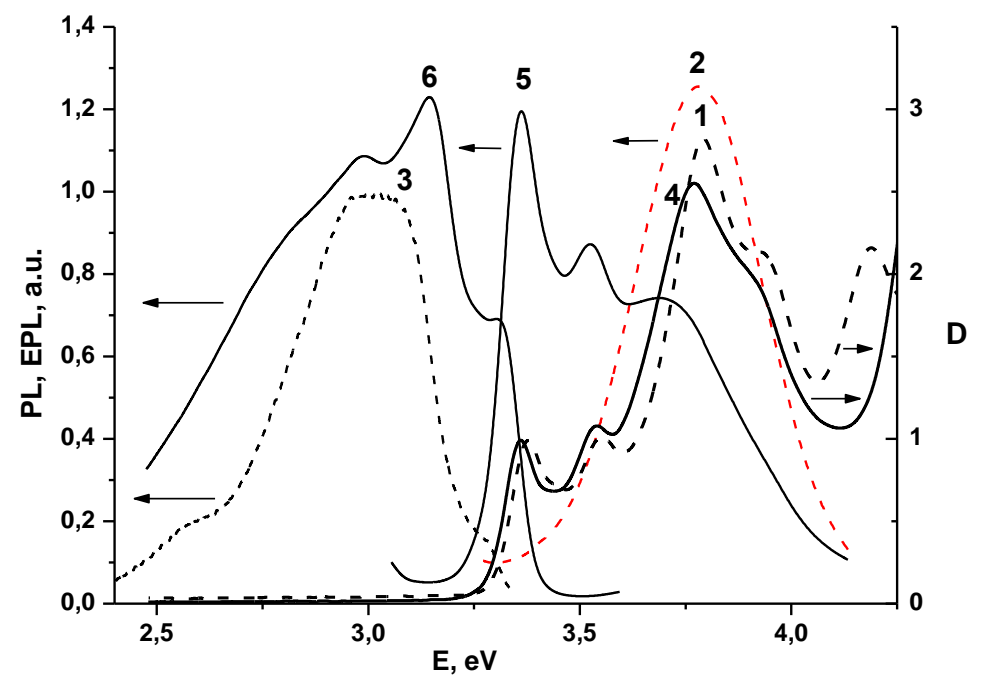

Fig. 3. Absorption spectra of solutions $(1,4)$ and photoluminescence excitation $(2,5)$ and photoluminescence $(3,6)$ spectra of films of V7BC (1-3) and V7BC-OMA (4-6) 
TABLE 2. Energies $\left(E_{\max }, e V\right)$ of Absorption and Photoluminescence Bands, Their Intensities Relative to the Strongest Band $\left(\mathrm{I} / \mathrm{I}_{1}\right)$, and Stokes Shift $(\mathrm{eV})$ for Films of VC Polymers and Copolymers Deposited onto a Quartz Substrate from Their $\mathrm{CHCl}_{3}$ Solutions

\begin{tabular}{|c|c|c|c|c|c|c|c|c|c|}
\hline \multicolumn{2}{|c|}{ PVC } & \multicolumn{2}{|c|}{ VC-OMA } & \multicolumn{2}{|c|}{ I-VC-OMA } & \multicolumn{2}{|c|}{ V7BC } & \multicolumn{2}{|c|}{ V7BC-OMA } \\
\hline $\mathrm{E}_{\max }$ & $\mathrm{I} / \mathrm{I}_{1}$ & $\mathrm{E}_{\max }$ & $\mathrm{I} / \mathrm{I}_{1}$ & $\mathrm{E}_{\max }$ & $\mathrm{I} / \mathrm{I}_{1}$ & $E_{\max }$ & $\mathrm{I} / \mathrm{I}_{1}$ & $\mathrm{E}_{\max }$ & $\mathrm{I} / \mathrm{I}_{1}$ \\
\hline \multicolumn{10}{|c|}{ Absorption } \\
\hline 3.60 & 1.00 & 3.61 & 1.0 & 3.52 & 1.00 & 3.38 & 0.35 & 3.35 & 0.39 \\
\hline \multicolumn{10}{|c|}{ Photoluminescence } \\
\hline & & 3.55 & 0.25 & & & & & 3.30 & 0.56 \\
\hline 3.26 & 0.70 & 3.34 & 1.00 & 3.32 & 0.88 & 3.0 & 1.0 & 3.14 & 1.00 \\
\hline 3.10 & 1.00 & 3.17 & 0.99 & 3.17 & 1.00 & 2.56 & 0.20 & 2.97 & 0.73 \\
\hline \multicolumn{10}{|c|}{ Stokes shift } \\
\hline & & 0.06 & & & & & & 0.05 & \\
\hline 0.34 & & 0.27 & & 0.21 & & 0.38 & & 0.21 & \\
\hline 0.50 & & 0.44 & & 0.35 & & 0.82 & & 0.38 & \\
\hline
\end{tabular}

The number of structure defects probably increased upon forming the V7BC fi $1 \mathrm{~m}$. Therefore, only two inflections appeared at 2.56 and $3.0 \mathrm{eV}$ near the excimer luminescence (Fig. 3). The PL spectra of I-VC-OMA and V7BC had similar shapes. However, the excimer energy in V7BC was $0.3 \mathrm{eV}$ less than that in I-VC-OMA because of the decreased interaction energy between the excimers in $\mathrm{V} 7 \mathrm{BC}$.

Steric hindrance to excimer formation in V7BC-OMA films decreased despite the high probability of forming structure defects from V7BC molecules during its copolymerization with OMA, which contained flexible spacers. As a result, another two maxima and two distinct inflections appeared in their PL spectra (Fig. 3). Apparently, these excimers had considerably different symmetries in the films because their emission occurred from the bottom of the excited band $(1 \mathrm{~A} \rightarrow 1 \mathrm{Lb})$ and as a result of $1 \mathrm{~A} \rightarrow 1 \mathrm{La}$-transitions. This was confirmed by the complicated PL excitation spectrum of V7BC-OMA films (Fig. 3) and the increased Stokes shift (Table 2), which depended on the polarization of the electronic transition. The latter indicated that the 
molecular ordering in films with long OMA spacers and substituents (iodine in I-VC-OMA and benzene ring in V7BC-OMA) was improved as compared with the analogs (PVC and V7BC) without OMA. This improved the structure of polymer aggregates in the films.

Conclusions. It was found that adding flexible OMA spacers to PVC via copolymerization increased substantially the solubility and ordering of the resulting PVC films as compared with films without OMA. This caused a slight narrowing of the absorption and PL excitation bands. The flexible OMA spacers in the PVC and V7BC polymer chain affected the energy of the excimer fluorescence band maxima. For example, excimer PL bands with maxima at 3.34 and $3.17 \mathrm{eV}$ were observed in spectra of VC-OMA films. These overlapped in spectra of PVC films without OMA so that only a broad PL band with a maximum at $3.10 \mathrm{eV}$ was observed.

Changes in all spectra that resulted from adding substituents to PVC depended strongly on their size (bulk). Thus, adding iodine caused mainly a significant red shift (77 meV) of the electronic ${ }^{1} \mathrm{~A}$ $\rightarrow{ }^{1} \mathrm{~L}_{\mathrm{b}}$-transition of polymer molecules polarized along the short axis. Adding a benzene ring increased substantially the intensities of ${ }^{1} \mathrm{~A} \rightarrow{ }^{1} \mathrm{~L}_{b}$-transitions of vinylcarbazole molecules polarized along the short axis and induced a large red shift of the maximum $(250 \mathrm{meV})$ for the ${ }^{1} \mathrm{~A} \rightarrow{ }^{1} \mathrm{~L}_{\mathrm{b}^{-}}$ transition of lowest energy. Furthermore, this substitution produced a blue shift in the energy of the PL excitation maximum near the absorption of ${ }^{1} \mathrm{~A} \rightarrow{ }^{1} \mathrm{~L}_{\mathrm{b}}$-transitions (by $530 \mathrm{meV}$ ).

The results could be used to develop new methods for increasing the photosensitivity of polymer composites over a broad spectral range.

\section{REFERENCES}

1. H. Hoegl, J. Phys. Chem., 69, No. 3, 755-766 (1965).

2. J. V. Grazulevicius, P. Strohriegl, J. Pielichowski, and K. Pielichowski, Prog. Polym. Sci., 28, No. 9, 1297-1353 (2003).

3. Ya. Vertsimakha and A. Verbitsky, in: Solar Cell Research Progress, J. Carson (Ed.), Nova Publ., New York (2008), Chap. 8, pp. 297-317.

4. Ya. Vertsimakha, A. Verbitsky, A. Ishchenko, V. Syromyatnikov, and I. Pomaz, Mol. Cryst. Liq. Cryst., 536, 99-106 (2011).

5. S. Studzinsky, V. Syromyatnikov, A. Ishchenko, N. Derevyanko, Ya. Vertsimakha, and A. Verbitsky, Nonlinear Opt., Quantum Opt., 33, Nos. 1-2, 151-159 (2005). 
6. Zh. Gao, Zh. Wang, T. Shan, Y. Liu, F. Shen, Y. Pan, H. Zhang, X. He, P. Lu, B. Yang, and Yu. Ma, Org. Electron., 15, 2667-2676 (2014).

7. Y. Liu, Sh. Lv, L. Li, and S. Shang, Synth. Met., 162, 1059-1064 (2012).

8. J. Li and A. C. Grimsdale, Chem. Soc. Rev., 39, 2399-2410 (2010).

9. A. Verbitsky, Ya. Vertsimakha, P. Lutsyk, S. Studzinsky, S. Bereznev, J. Kois, A. Opik, and E. Mellikov, Proc. Estonian Acad. Sci. Chem., 55, No. 2, 111-119 (2006).

10. B. Jezek et al., Czech. J. Phys. B, 25, 1176-1180 (1975).

11. V. M. Yashchuk, Photonics of Polymers [in Ukrainian], Kyiv. Univ., Kyiv (2004).

12. Y. Nozue, T. Hisamune, T. Goto, and H. Tsuruta, J. Phys. Soc. Jpn., 52, 2983-2985 (1983). 